\title{
Patient-Related Benefits for Adults with Cochlear Implantation: A Multicultural Longitudinal Observational Study
}

\author{
Thomas Lenarz $^{\mathrm{a}}$ Lida Muller $^{\mathrm{b}}$ Hanna Czerniejewska-Wolska ${ }^{\mathrm{c}}$ \\ Hector Vallés Varela ${ }^{g}$ César Orús Dotúc Marcin Durko ${ }^{d}$ Alicia Huarte Irujo ${ }^{i}$ \\ Bartosz Piszczatowski $^{\mathrm{e}}$ Marek Zadrożniak ${ }^{f}$ Colin Irwin $^{j}$ Petra L. Graham ${ }^{k}$ \\ Josie Wyss ${ }^{j}$

\begin{abstract}
${ }^{a}$ Medizinische Hochschule Hannover, Hannover, Germany; ${ }^{b}$ Tygerberg Hospital, Stellenbosch University Cochlear Implant Unit, Tygerberg, South Africa; ' Department of Phoniatrics and Audiology, Poznań University of Medical Sciences, Poznań, dDepartment of Otolaryngology and Laryngological Oncology, Medical University of Lodz, Barlicki University Hospital, Lodz, e'Department of Otolaryngology, Medical University of Bialystok, Bialystok, and ${ }^{\mathrm{f}}$ Department of Otolaryngology and Laryngological Oncology, Medical University of Lublin, Lublin, Poland; ${ }^{9}$ Hospital Clinico Universitario Lozano Blesa, Zaragoza, hFundacion de Gestion Sanitaria, Hospital de Sant Pau, Barcelona, and 'Clinica Universitaria de Navarra, Pamplona, Spain; ${ }^{j}$ Cochlear AG, Basel, Switzerland; ${ }^{2}$ Department of Statistics, Macquarie University, Sydney, NSW, Australia
\end{abstract}

\section{Keywords}

Observational study - Patient registry - Quality of life .

Hearing outcomes - Patient-related benefits · Health utility . Hearing benefits - Speech, Spatial, and Qualities of Hearing Scale $\cdot$ Health Utilities Index Mark 3

\begin{abstract}
Objectives: To assess subjectively perceived, real-world benefits longitudinally for unilateral cochlear implant $(\mathrm{Cl})$ recipients in a multinational population treated routinely. To identify possible predictors of self-reported benefits. Design: This was a prospective, multicenter, repeated-measures study. Self-assessment of performance at preimplantation and postimplantation at 1, 2, and 3 years using standardized, validated, local language versions of the Speech, Spatial, and Qualities of Hearing Scale (SSQ), and the Health Utilities Index Mark 3 (HUI3) was performed. Outcomes were analyzed us-
\end{abstract}

\section{KARGER}

E-Mail karger@karger.com www.karger.com/aud
(C) 2017 The Author(s)

Published by S. Karger AG, Basel

This article is licensed under the Creative Commons AttributionNonCommercial-NoDerivatives 4.0 International License (CC BYNC-ND) (http://www.karger.com/Services/OpenAccessLicense) tribution of modified material requires written permission. ing a longitudinal mixed-effects model incorporating country effect. Patient demographics were explored for associations with change over time. Subjects: Two hundred ninetyone routinely treated, unilateral $\mathrm{Cl}$ recipients, aged 13-81 years, from 9 clinics across 4 countries. Results: Highly significant improvements were observed for all outcome measures $(p<0.0001)$. Postimplantation, mean outcome scores remained stable beyond 1 year, with notable individual variability. A significant association for one or more outcomes with preimplantation contralateral hearing aid use, telephone use, age at implantation, implantation side, preimplantation comorbidities, dizziness, and tinnitus was observed ( $p<0.004)$. Conclusions: Longitudinal benefits of $\mathrm{Cl}$ treatment can be measured using clinically standardized selfassessment tools to provide a holistic view of patient-related benefits in routine clinical practice for aggregated data from multinational populations. Self-reported outcomes can provide medical-based evidence regarding $\mathrm{Cl}$ treatment to support decision-making by health service providers.

(C) 2017 The Author(s)

Published by S. Karger AG, Basel

Josie Wyss

Cochlear AG

Peter-Merian-Weg 4

CH-4052 Basel (Switzerland)

E-Mail jwyss@ cochlear.com 


\section{Introduction}

The increasing demand for access to reliable, largescale, longitudinal, patient-related outcome data on routine clinical treatment, both in and out of the field of cochlear implants (CI), underlines a growing need for greater universal consistency in the monitoring of treatment benefits, and the data collection methods that can serve as medical evidence for the respective therapies [Gliklich and Dreyer, 2010; Haute Autorité de Sauté, 2011; National Institute for Health and Clinical Excellence, 2013; US Food and Drug Administration, 2013a, b; World Medical Association, 2013]. Such clinical evidence allows various stakeholders, including potential candidates, treatment recipients, health care professionals, market regulators, and health policy makers, to make well-informed decisions on their effectiveness, provision, and reimbursement.

In the last decade, various health technology assessment appraisals of hearing implants have been performed, resulting in guidelines for health practices relating to their provision in adults and children in the UK, France, and the USA [National Institute for Health and Clinical Excellence, 2009; Haute Autorité de Santé, 2011; Tufts Evidence-Based Practice Centre under contract by the Agency for Health Research and Quality, 2011]. In consideration of the available published evidence for the appraisals, the reports call for clinical research publications to include greater transparency of the study methods and measures used, greater consistency across studies, larger treatment cohorts, more detail regarding the cohort demographics, longer-term follow-up, treatment controls, and appropriate outcome measures that also reflect patient-related benefits in the real world [National Institute for Health and Clinical Excellence, 2009; Tufts EvidenceBased Practice Centre under contract by the Agency for Health Research and Quality, 2011].

The use of multicenter patient registries on a national level has the potential to yield large amounts of data and to improve the efficiency of the data collection process; however, it is acknowledged that there are challenges associated with the high costs of the initial setup and maintenance and the need for adaptation of clinical practices in order for them to be adopted [Berrettini et al., 2011]. Successful examples of nationally based registries to capture CI patient outcomes, based on standard audiological measures, have been implemented and sustained in the long term both in France and Switzerland, involving all implanting CI clinics, all commercially available CIs, and recipients of all ages [Merrina, unpubl. data; Brand et al.,
2014]. With the number of enrolled CI recipients exceeding several thousands, they provide credible evidence of the safety and clinical effectiveness of CI treatment in routine care. Nonetheless, there is little or no insight into patient-related benefits in the real world.

In September 2011, the Cochlear Implant Recipient Observational Study (IROS), an international, voluntary, observational study of the patient-related benefits obtained in routine therapy, was initiated by Cochlear LTD (Sydney, NSW, Australia). Implemented via an electronic platform to facilitate consistent data collection, the ongoing study includes 2 main outcome measures: the Speech, Spatial, and Qualities of Hearing Scale (SSQ), an established disease-specific measure of daily hearing function, and the Health Utilities Index Mark 3 (HUI3), a widely used, generic measure of health utility gains [Feeny et al., 1995; Noble and Gatehouse, 2004].

The aim of this study was to determine real-life treatment benefits among unilateral CI treatment recipients from a multinational cohort in routine care, via repeated measures of the HUI3 and the SSQ. Furthermore, the assessment of recipient variables as potential predictors of change in outcomes over time was undertaken.

To our knowledge, this is the first report of a collaborative, international effort to collect, aggregate, and describe longitudinal self-reported patient-related benefits for unilateral CI recipients in routine care.

\section{Methods}

This prospective observational study had a repeated measures design and was conducted in accordance with the Declaration of Helsinki (ethical principles for medical research involving human subjects) and international guidelines for the conduction and reporting of clinical investigations [International Organization for Standardization, 2011; World Medical Association, 2013]. Ethics committee approval for study participation was obtained for each participating clinic as required at the national or local level. The outline of the Cochlear IROS and the list of approving ethics committees are available for public access (ClinicalTrials \#NCT2004353, https://clinicaltrials.gov).

Formal written informed consent was obtained from each recipient prior to their enrolment into this study. Clinics were advised to approach patients in chronological order, following their decision to implant any hearing device from the company Cochlear Ltd., for consent to participate in the study. Patients were approached between the day of surgery and the first switch-on of their external sound processor. In the case of adolescents (age 1017 years), their carer was approached for consent.

\section{Participating Sites}

For inclusion in this study, clinics participating in the study for $\geq 2$ years that had enrolled $\geq 10$ CI recipients with longitudinal data
Lenarz et al. 
Fig. 1. Global registration of hearing implant recipients with all device types and configurations as enrolled across 30 participating implant sites and the subset of 291 unilateral cochlear implant (CI) recipients included in this study enrolled at the 9 coauthor clinics (data extraction date: October 30,2015$)$.

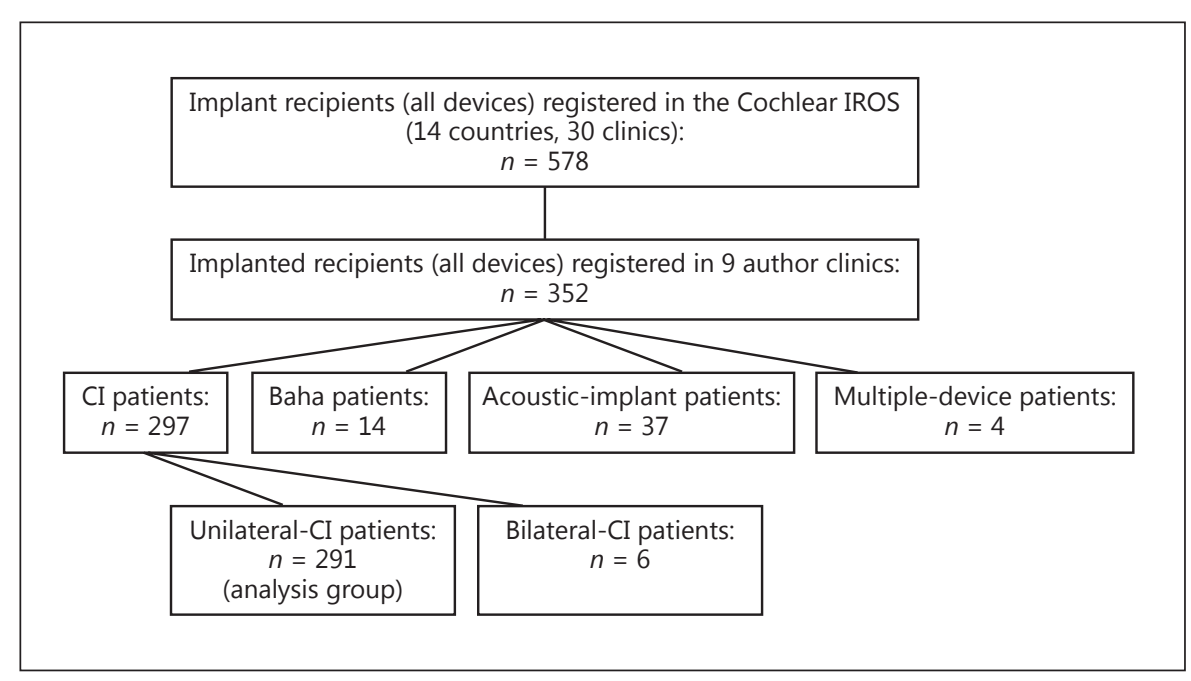

at the time of data extraction (October 2015) were approached to share their data for collective analysis. All 9 implant centers approached agreed to participate. This included 1 German, 1 South African, 3 Spanish, and 4 Polish clinics. All available data for the respective 291 enrolled unilateral CI recipients were included in the analysis (Fig. 1).

\section{Main Outcome Measures}

Health Utilities Index 3: The "4-week recall" version of the selfassessed HUI3 with 15 questions was completed at each assessment interval. Licensed versions in English, Spanish, German, and Polish were obtained from the developers, Health Utilities Inc., while an Afrikaans language version was developed for this study and shared with the developers. In this generic health-related quality of life questionnaire, the responder is asked to assess their perceived degree of impairment in each of 8 subdomains of health: vision, hearing, speech, ambulation, dexterity, emotion, cognition, and pain [Feeny et al., 1995, 2002]. A multiattribute health utility score is derived that enables classification of the respondent's overall health status into predefined health states ranging from -0.36 to 1.00 , where a negative score represents a state "worse than dead" and a top score of 1.00 is perfect health. A significant change in health utility gain between assessment intervals of 0.03 was used [Feeny et al., 2002]. The HUI3 was selected over other global health utility measures available as it is considered to be the most sensitive measure for effects of hearing treatment on overall health status [UK Cochlear Implant Study Group, 2004; Damen et al., 2007; Maes et al., 2011].

The Speech, Spatial, and Qualities of Hearing Scale: The SSQ with 49 questions divided into 3 subcategories was used for selfassessment of hearing function at each interval. The SSQ is a standardized, hearing disease-specific questionnaire in which the responder is asked to assess their hearing ability with reference to a variety of realistic, real-world listening situations [Gatehouse and Noble, 2004]. The SSQ was chosen as it has been reported to be sensitive to changes in hearing function following treatment with a variety of hearing therapies, implantable and nonimplantable hearing devices, and stimulation configurations [Noble and Gatehouse, 2006; Noble et al., 2008; Laske et al., 2009; House et al., 2010;
Noble, 2010; Pai et al., 2012; Mertens et al., 2015]. While many versions of the SSQ exist, the English version 5.6 with 49 questions, available online from the British Medical Research Council, was used as a master version for subsequent validated translations to German, Spanish, Polish, and Afrikaans.

Each question was scored on a 21 -point rating scale ranging from 0 to 10 , corresponding to minimum and maximum hearing abilities, respectively. Subsequently, subcategory mean scores were derived by averaging responses across all questions within each category. Clinically significant changes in subcategory scores between assessments were set at $\geq 1.0$ [Noble and Gatehouse, 2006].

\section{Translations for Evaluation Tools}

All case report forms and outcome measures completed by the implant recipient were provided in their native language. Clinician questionnaires were also provided in the local native language and in English for clinicians in South Africa. While the case report forms used to gather hearing profile and demographic information followed a 1-way translation process of the English master version to each respective language, both main outcome scales, i.e., HUI3 and SSQ, were translated via the recommended validated translation process by the respective scale developers.

The process of validation of the translation of SSQ version 5.6 (available through the British Medical Research Council) was a forward and backward validation translation process involving professional certified translators and proofers. In brief: the SSQ was translated from English to the required native language by translator 1; the new native-language version was then translated backward to English by translator 2; an English proofer then compared the English backward-translated version to the original English master version, identifying and annotating discrepant context or content for further address. Annotations were addressed and incorporated by translator 1 to finalize the new language version.

The process of validation of the translation for the HUI3 4-week recall version was documented and controlled by the licensers and developers of the HUI3 (HUI Inc.). In summary, this involved a validation translation process requiring 2 forward-translation versions from English to the new language by translator 1 and translator 2 in parallel. A third proofer/translator decided on the best 
Table 1. Demographics of the 291 enrolled unilaterally implanted cochlear implant recipients

\begin{tabular}{|c|c|c|c|}
\hline \multicolumn{2}{|l|}{ Gender } & \multicolumn{2}{|l|}{ Implant ear } \\
\hline Female & $163(56)$ & Left & $127(44)$ \\
\hline Male & $128(44)$ & Right & $164(56)$ \\
\hline Age at implantation & & Hearing loss degree & \\
\hline$<18$ years & $6(2)$ & Moderate-severe & $33(11)$ \\
\hline $18-64$ years & $223(77)$ & Profound & $153(53)$ \\
\hline $65+$ years & $62(21)$ & NA & $105(36)$ \\
\hline Progression/onset & & Tinnitus & \\
\hline Progressive & $196(67)$ & No & $102(35)$ \\
\hline Sudden & $75(26)$ & Yes & $151(52)$ \\
\hline NA & $20(7)$ & NA & $38(13)$ \\
\hline Hearing loss type & & Comorbidities & \\
\hline Mixed/other & $9(3)$ & No & $253(87)$ \\
\hline Sensorineural & $169(58)$ & Yes & $22(8)$ \\
\hline NA & $113(39)$ & NA & $16(5)$ \\
\hline Preimplantation HA & & Dizziness & \\
\hline Bilateral & $115(40)$ & Never & $124(43)$ \\
\hline Unilateral, either ear & $60(21)$ & Sometimes & $99(34)$ \\
\hline Unaided & $103(35)$ & Half the time or more & $30(10)$ \\
\hline NA & $13(4)$ & NA & $38(13)$ \\
\hline Employment & & Telephone use & \\
\hline Full time & $124(43)$ & Yes & $143(49)$ \\
\hline Part time & $16(5)$ & No & $130(45)$ \\
\hline Retired & $76(26)$ & NA & $18(6)$ \\
\hline Unemployed & $57(20)$ & Country & \\
\hline NA & $18(6)$ & Germany & $56(19)$ \\
\hline Duration of HL & & Poland & $98(34)$ \\
\hline Mean \pm SD: $22.6 \pm 15.4$ years & $256(88)$ & South Africa & $87(30)$ \\
\hline NA & $35(12)$ & Spain & $50(17)$ \\
\hline
\end{tabular}

Values are presented as $n(\%)$. NA, not available/not answered; HA, hearing aid; HL, hearing loss.

version per question/phrase and compiled the final validated translation version. (Note: to enable recipients to respond in their native language for self-assessment, validated translations were implemented for: Afrikaans, Arabic, Danish, Dutch, English, French, German, Hebrew, Hungarian, Japanese, Polish, Portuguese [Brazilian and European versions], Slovenian, Spanish, Swedish, and Turkish.)

\section{Evaluation Schedule}

The evaluation intervals for baseline (preimplantation status) and postimplantation at 1,2, and 3 years were adopted to coincide with routine annual clinical follow-up visits (i.e., \pm 2 months at each expected annual visit date). Preimplantation assessment was performed following the decision of implant treatment and device type and configuration between the day of surgery and before the first switch-on of the sound processor. This was to avoid the influence of the new hearing sensation via the hearing implant system for baseline self-assessment of the preimplantation hearing and health status. At each assessment interval, a 4-week recall period was referenced, i.e., " 4 weeks prior to implant surgery" for baseline and "within the last 4 weeks" at each postimplantation interval.

HUI3 and SSQ self-assessments were completed by the recipient either on paper (usually in the clinic or at home) or directly online depending their choice upon consent to participate. Data entry was performed from the completed paper forms by a representative of the clinic. By study design, and through the use of autoprompts for online data entry, $100 \%$ completion of the HUI3 and at least 45 out of 49 questions for the SSQ were requested as required for analysis.

\section{Subject Inclusion}

Any routinely treated hearing-impaired adult or adolescent ( $>10$ years old), meeting local criteria for hearing implant treatment with any hearing implant from the company Cochlear was eligible for enrolment (Fig. 1). Only data for enrolled unilateral CI recipients were included in this study. At online enrolment, a subject study code was automatically assigned to each recipient, thus maintaining their full anonymity on the study platform. Patient demographics including medical and hearing histories were collected both via the clinician from the patient hospital file and via the recipient directly at enrolment using the respective case report forms (Table 1). However, responses to individual demographic questions on these case report forms were not compulsory, thus allowing for missing responses.
$64 \quad$ Audiol Neurotol 2017;22:61-73
Lenarz et al. 
Statistical Analysis

The aim of the data analysis reported in this paper is to address the following questions:

1. How do outcomes for HUI3 and SSQ scores change over time for the unilateral CI cohort?

2. What is the nature of the missing data at follow-up intervals and the impact on outcomes?

3. Are preimplantation patient variables associated with longitudinal outcomes for SSQ and HUI3?

Analysis of all of the available data extracted from the central database, the Cochlear IROS, on October 30, 2015, was undertaken using $\mathrm{R}$ version 3.2.0 [R Core Team, 2015]. Statistical analysis was performed by a coauthor (consultant statistician at Macquarie University, Sydney, Australia).

For description of the data, a summary of continuous variables using means and SD or SE and of categorical variables using numbers per subgroup and percentage of the total study cohort was created.

Examination of the missing outcome data and their impact on cohort outcomes was undertaken to explore the potential for informative missing status. Outcomes for recipients with subsequent missing follow-up data were compared to outcomes for recipients with no missing data. In this context, informative missing status would result if the reason for dropout were related to hearing performance as opposed to random dropout that might occur due to work, vacation, or non-hearing-related illness. The mean outcomes for subgroups with missing data and no missing data were compared using Welch 2-sample $t$ tests to determine whether systematic differences between the subgroups existed that might suggest evidence of nonrandom missing status.

Linear mixed-effects models were used to model changes over time with a nested random intercept for recipient within country to control for the correlation induced through repeated measures on the same recipients and to control for country effects. Tukey pairwise differences were used to compare all pairs of time points. Linear mixed-effects models provide unbiased estimates of effects in the presence of randomly missing responses [Little and Rubin, 2002].

To determine whether multicultural subgroups differed in their response on HUI3 and SSQ subcategories, the variable country was initially incorporated as a predictor into the linear mixedeffects model with a random intercept for recipient using an interaction between country and assessment time point. A random intercept for country (with recipient nested within country) was then incorporated into the model to control for significant countrytime interactions.

Interactions between time of assessment and 12 recipient variables collected at baseline were examined for their association with HUI3 and SSQ subcategory scores. If the interaction with time was not significant, the main effects were examined. A Bonferroni type correction to the significance level was used to control for the 12 recipient variables assessed for each outcome measure. As such, an interaction or main effect was considered significant for a modified $p$ value $<0.004(0.05 / 12)$. For significant interactions in categorical variables with more than 2 groups, Tukey's pairwise multiple comparisons were used to determine which groups differed at which time points. Postimplantation variables were not explored for their impact on outcomes for this study.

Patient-Related Benefits for Adults with

Cochlear Implantation

\section{Results}

\section{Enrolled Implant Population}

At the time of data extraction, a total of 578 implant recipients with a variety of hearing implants had been enrolled by more than 30 international collaborating clinics. The 9 coauthor implant clinics enrolled 352 recipients, 297 of whom were CI recipients (291 unilateral implantees and 6 sequential bilateral CI users) (Fig. 1).

\section{Study Cohort Demographics}

Table 1 illustrates the summary demographics for the study cohort of 291 unilateral CI recipients. The national contributions of the recipients were as follows: about one third were from Poland and South Africa and about one fifth were from Germany and Spain. The cohort comprised slightly more female than male recipients. The mean age of the cohort was 50.1 years (SD 17.4, range 13-91), the majority (i.e., about four fifths) were aged 18 64 years, about one fifth of the subjects were aged $\geq 65$ years at implantation, and 6 were of adolescent age (range 13-17 years). The duration of hearing loss, reported for $88 \%$ of the cohort, was 22.6 years on average (SD 15.4, range $0-67$ ), while no response was provided by 12 recipients.

Approximately half of the recipients presented with right-sided implants, and more than two thirds had a progressive onset of hearing loss. Hearing loss degree and type of hearing loss in the implant ear, reported for two thirds of the cohort, were sensorineural and of a profound degree for the vast majority.

Prior to implantation, approximately equal sized subgroups reported experiencing dizziness versus never experiencing it (i.e., 44 and $43 \%$, respectively), while $13 \%$ did not respond. Tinnitus was experienced by about half of the subjects and it was never experienced by about one third of the subjects, while $13 \%$ of the cohort did not respond. Comorbidities were absent in the vast majority of patients (i.e., $87 \%$ ) and present in $8 \%$ of the cohort; no response was provided for $5 \%$ of the group.

Preimplantation acoustic amplification was used by $61 \%$ of the cohort in either a bilateral $(40 \%)$ or a unilateral $(21 \%)$ configuration. More than one third (35\%) of the subjects reported that they did not use amplification immediately prior to implant surgery, while no response was available for $4 \%$ of the patients. Not shown in Table 1 , the daily hearing aid use on the implant side ( $n=$ 174) averaged $13.7 \mathrm{~h}$ (SD 4.3) and 13.9 $\mathrm{h}$ (SD 4.1) for the contralateral ear $(n=174)$, ranging between 0 and $24 \mathrm{~h}$ for each ear. Approximately equal subgroups reported

Audiol Neurotol 2017;22:61-73

DOI: $10.1159 / 000477533$ 
Table 2. Estimated SSQ and HUI3 outcomes at each visit with $p$ value for the overall test of a change between one or more pairs of evaluation time points

\begin{tabular}{lllllll}
\hline Outcome & $n$ & Baseline & $\begin{array}{l}\text { 1 year } \\
\text { postimplantation }\end{array}$ & $\begin{array}{l}\text { 2 years } \\
\text { postimplantation }\end{array}$ & $\begin{array}{l}3 \text { years } \\
\text { postimplantation }\end{array}$ \\
\hline HUI3 multiattribute & 282 & $0.457(0.015)$ & $0.606(0.018)$ & $0.600(0.022)$ & $0.540(0.038)$ \\
SSQ speech & 276 & $2.197(0.201)$ & $4.553(0.212)$ & $4.597(0.236)$ & $5.006(0.338)$ & $<0.001$ \\
SSQ spatial & 276 & $2.357(0.125)$ & $4.616(0.144)$ & $4.616(0.180)$ & $4.883(0.306)$ & $<0.001$ \\
SSQ qualities & 274 & $3.380(0.210)$ & $5.508(0.221)$ & $5.600(0.249)$ & $6.311(0.359)$ & $<0.001$ \\
\hline
\end{tabular}

Values are presented as means (SE) unless otherwise stated. SSQ, Speech, Spatial, and Qualities of Hearing Scale; HUI3, Health Utilities Index Mark 3.

Table 3. Baseline data for all registered unilateral cochlear implant recipients for HUI3 and SSQ subcategories with missing and not missing follow-up data and $p$ values from Welch 2 -sample $t$ tests

\begin{tabular}{|c|c|c|c|c|c|}
\hline & \multicolumn{2}{|c|}{ Not missing at 1 year postimplantation } & \multicolumn{2}{|c|}{ Missing at 1 year postimplantation } & \multirow[t]{2}{*}{$p$ value } \\
\hline & $n$ & mean (SD) & $n$ & mean (SD) & \\
\hline HUI3 multiattribute & 201 & $0.453(0.244)$ & 81 & $0.469(0.279)$ & 0.647 \\
\hline SSQ speech & 195 & $2.175(1.787)$ & 81 & $2.249(2.010)$ & 0.774 \\
\hline SSQ spatial & 195 & $2.411(1.974)$ & 81 & $2.237(1.956)$ & 0.503 \\
\hline SSQ qualities & 194 & $2.311(2.148)$ & 80 & $3.380(2.397)$ & 0.922 \\
\hline
\end{tabular}

SSQ, Speech, Spatial, and Qualities of Hearing Scale; HUI3, Health Utilities Index Mark 3.

that they could or could not use the telephone (i.e., 49 and $45 \%$, respectively), and $6 \%$ did not respond. Active employment was reported by about half of the recipients; about one fourth of the subjects were retired, about one fifth were unemployed, and 6 recipients did not reply.

Not shown in Table 1, the most frequently reported etiology for the implant ear was unknown etiology $(n=$ $109 ; 37 \%)$, followed by familial $(n=22 ; 8 \%)$, otosclerosis $(n=15 ; 5 \%)$, meningitis $(n=12 ; 4 \%)$, ototoxic drugs $(n=$ $11 ; 4 \%)$, and noise exposure $(n=11 ; 4 \%)$. Many other etiologies made up the remaining 38\%. Etiology was not considered for further analysis.

Postimplantation, all enrolled unilateral CI recipients were implanted with Nucleus ${ }^{\circledR}$ CI (Cochlear LTD), including the Nucleus ${ }^{\circledR}$ Freedom CI24RE (CA) and the Nucleus ${ }^{\circledR}$ CI422 for the vast majority, followed by the Nucleus ${ }^{\circledR}$ CI512. Four patients were implanted each with the Nucleus ${ }^{\circledR} 24$ Contour Advance ${ }^{\circledR}$ and with the Nucleus ${ }^{\circledR}$ Hybrid-CI24REH. Implant model was not considered for further analysis. At the time of data extraction, the time since surgery was on average 2.6 years (SD 1.1, range $0.2-$ 4.9).

At 1 year postimplantation, the majority of the unilateral CI recipient study cohort (i.e., 91\%; 183/201) presented as all-day implant users, with a mean daily use of 14.1 $\mathrm{h}$ (SD 3.3, range 3-24). In addition, contralateral hearing aid use was reported by $50 \%$ of the cohort $(100 / 201)$.

\section{Baseline Performance Outcome Data}

The mean SSQ and HUI3 outcomes for the group at baseline for the preimplantation condition are shown in Table 2, along with the SE. HUI3 multiattribute health utility values ranged from -0.197 to 1.00 for the individual, with a cohort mean of 0.46 . SSQ subcategory cohort mean scores ranged from 2.2 to 3.4, and those for the individual ranged from 0 to 10 .

Baseline mean scores for enrolled unilateral CI recipients with missing 1-year follow-up data at the time of data extraction (27\% of recipients) with mean scores for recipients who had 1-year postimplantation follow-up data are compared and shown in Table 3. Two-sample $t$ tests 


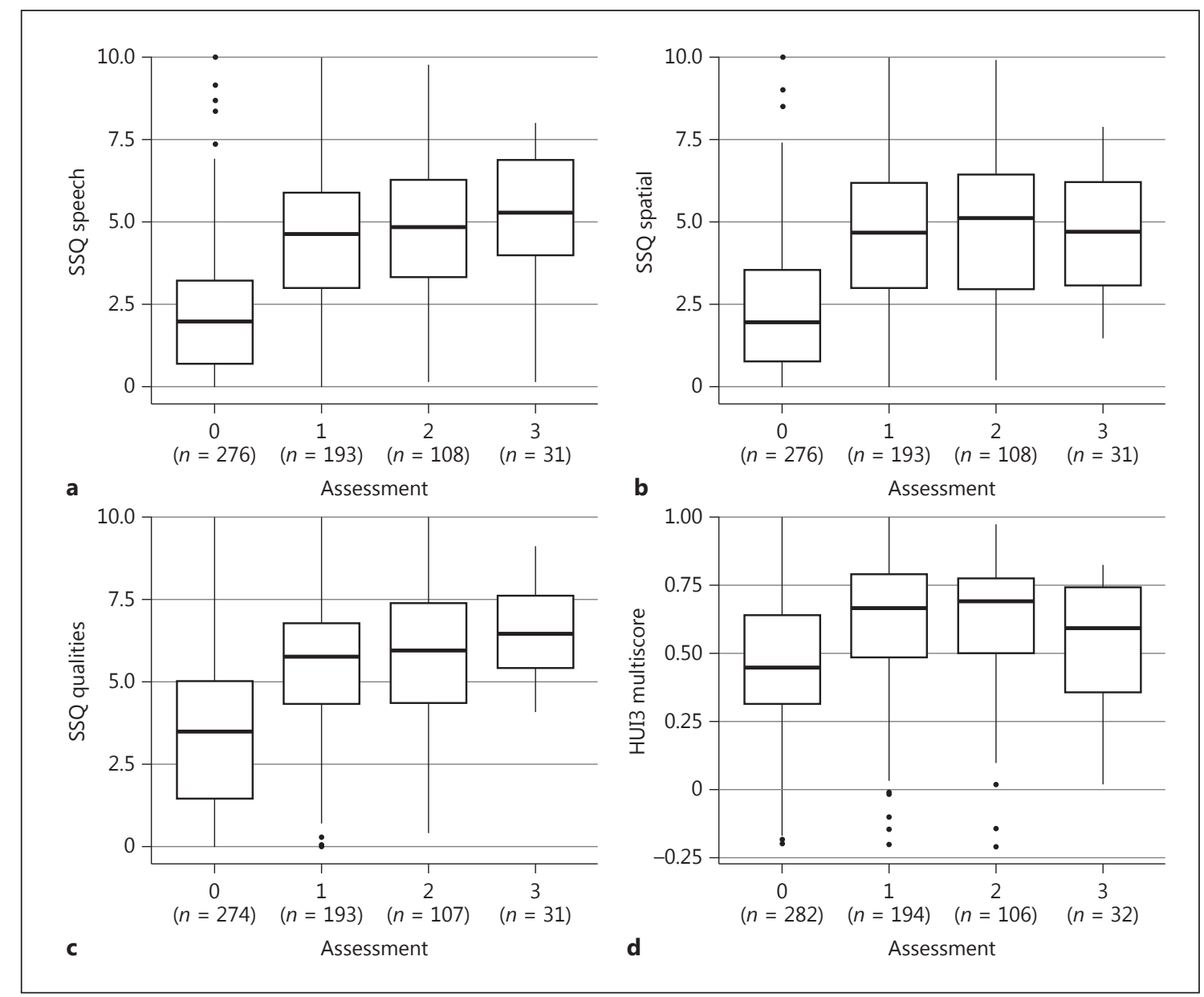

Fig. 2. Box-and-whisker plots showing the distribution of self-assessment outcome scores for Speech, Spatial, and Qualities of Hearing Scale (SSQ) subcategories speech (a), spatial (b), and qualities (c) and for Health Utilities Index Mark 3 (HUI3) multiattribute utility (d). All available data sets at each assessment interval

suggest no evidence of a difference between mean outcome responses for the subgroup with missing data versus the subgroup with no missing data for all measures $(p>0.50)$. Similar results were seen when comparing data at 1-year postimplantation for the subgroup with missing data versus the subgroup with no missing data at the second year postimplantation and the third year postimplantation ( $p>0.07$, results not presented). Hence, there is no evidence of a systematic difference between outcomes for subjects with missing data and those with no missing data at subsequent follow-up intervals. As such, the assumption is that, at the time of the data extraction, the missing data were missing at random for non-hearing-related reasons.

Patient-Related Benefits for Adults with Cochlear Implantation are shown for the 291 subjects enrolled in the unilateral cochlear implant recipient cohort. Test intervals represent: baseline (preimplantation), i.e., 0 , and annual follow-up at 1, 2, and 3 years postimplantation.

The interaction between visit and country upon outcomes was observed to be significant for the SSQ subcategories speech $(p=0.022)$, spatial $(p=0.002)$ and qualities $(p<0.001)$, indicating that the effect of country should be controlled for in the remaining analyses. In contrast, no statistically significant country effect was observed for the HUI3 multiattribute score either as an interaction with time $(p=0.433)$ or as a main effect $(p=0.050)$.

\section{Longitudinal Performance Outcomes}

The distribution of outcome scores for the available data at each time point for each outcome measure is presented in Figure 2. Median scores suggest an increase from baseline and stability from 1-year postimplantation 
Table 4. Pairwise comparisons showing difference scores between visits for cochlear implant recipients

\begin{tabular}{|c|c|c|c|c|c|c|c|c|c|c|c|c|}
\hline \multirow{2}{*}{$\begin{array}{l}\text { Postimplantation } \\
\text { change }\end{array}$} & \multicolumn{3}{|c|}{ SSQ speech } & \multicolumn{3}{|c|}{ SSQ spatial } & \multicolumn{3}{|c|}{ SSQ qualities } & \multicolumn{3}{|c|}{ HUI3 multiattribute } \\
\hline & change & SE & $p$ value & change & SE & $p$ value & change & SE & $p$ value & change & SE & $p$ value \\
\hline 1 year - BSL & 2.356 & 0.139 & $0.000^{*}$ & 2.258 & 0.143 & $0.000^{*}$ & 2.128 & 0.150 & $0.000^{*}$ & 0.149 & 0.018 & $0.000^{*}$ \\
\hline 2 year - BSL & 2.400 & 0.175 & $0.000^{*}$ & 2.258 & 0.180 & $0.000^{*}$ & 2.220 & 0.189 & $0.000^{*}$ & 0.143 & 0.022 & $0.000^{*}$ \\
\hline 3 year - BSL & 2.809 & 0.297 & $0.000^{*}$ & 2.526 & 0.306 & $0.000^{*}$ & 2.931 & 0.320 & $0.000^{*}$ & 0.082 & 0.038 & 0.118 \\
\hline 2 year -1 year & 0.044 & 0.180 & 0.994 & 0.000 & 0.185 & 1.000 & 0.091 & 0.194 & 0.963 & -0.006 & 0.023 & 0.993 \\
\hline 3 year -1 year & 0.453 & 0.300 & 0.416 & 0.268 & 0.309 & 0.813 & 0.802 & 0.323 & 0.057 & -0.067 & 0.038 & 0.283 \\
\hline 3 year -2 year & 0.409 & 0.309 & 0.532 & 0.267 & 0.317 & 0.826 & 0.711 & 0.333 & 0.132 & -0.060 & 0.039 & 0.400 \\
\hline
\end{tabular}

BSL, baseline. ${ }^{*} p<0.05$.

Table 5. Significance of the interaction between predictor and visit and main effect of the predictor (Bonferroni type correction for multiple analyses)

\begin{tabular}{|c|c|c|c|c|c|c|c|c|}
\hline \multirow[t]{2}{*}{ Covariate } & \multicolumn{2}{|c|}{ HUI3 multiattribute } & \multicolumn{2}{|c|}{ SSQ speech } & \multicolumn{2}{|c|}{ SSQ spatial } & \multicolumn{2}{|c|}{ SSQ qualities } \\
\hline & $\begin{array}{l}\text { inter- } \\
\text { action }\end{array}$ & $\begin{array}{l}\text { main } \\
\text { effect }\end{array}$ & $\begin{array}{l}\text { inter- } \\
\text { action }\end{array}$ & $\begin{array}{l}\text { main } \\
\text { effect }\end{array}$ & $\begin{array}{l}\text { inter- } \\
\text { action }\end{array}$ & $\begin{array}{l}\text { main } \\
\text { effect }\end{array}$ & $\begin{array}{l}\text { inter- } \\
\text { action }\end{array}$ & $\begin{array}{l}\text { main } \\
\text { effect }\end{array}$ \\
\hline Comorbidities & 0.630 & $<0.001$ & 0.487 & 0.475 & 0.412 & 0.244 & 0.402 & 0.905 \\
\hline Gender & 0.061 & 0.684 & 0.665 & 0.162 & 0.630 & 0.746 & 0.544 & 0.390 \\
\hline Age group (<65 vs. $65+$ years) & 0.027 & 0.367 & 0.707 & 0.004 & 0.753 & 0.236 & 0.132 & 0.351 \\
\hline Hearing loss degree & 0.925 & 0.650 & 0.545 & 0.663 & 0.021 & 0.211 & 0.042 & 0.730 \\
\hline Implant side & 0.141 & 0.832 & 0.002 & 0.098 & 0.586 & 0.884 & 0.336 & 0.777 \\
\hline Onset of deafness & 0.755 & 0.732 & 0.338 & 0.047 & 0.017 & 0.058 & 0.263 & 0.022 \\
\hline Duration of deafness & 0.124 & 0.963 & 0.601 & 0.077 & 0.818 & 0.427 & 0.535 & 0.062 \\
\hline Telephone use Pre & $<0.001$ & $<0.001$ & 0.302 & $<0.001$ & 0.004 & $<0.001$ & $<0.001$ & $<0.001$ \\
\hline Contralateral HA Pre & $<0.001$ & 0.901 & 0.131 & 0.154 & 0.006 & 0.687 & $<0.001$ & 0.901 \\
\hline Employment Pre & 0.590 & 0.008 & 0.202 & 0.012 & 0.995 & 0.031 & 0.902 & 0.009 \\
\hline Tinnitus Pre & 0.132 & 0.001 & 0.042 & 0.002 & 0.066 & 0.012 & 0.225 & 0.001 \\
\hline Dizziness Pre & 0.367 & $<0.001$ & 0.342 & 0.210 & 0.280 & 0.334 & 0.188 & 0.319 \\
\hline
\end{tabular}

Significant $p$ values are in bold. $p<0.004(0.05 / 12)$ was considered statistically significant. Pre, preimplantation; HA, hearing aid.

onwards. At each time point a wide range of scores were observed. Estimated mean outcome scores and SE are shown for each outcome measure at each assessment interval in Table 2. Analysis of change over time suggests a highly statistically significant difference in group scores between at least one pair of assessments for all outcome measures $(p<0.001)$.

Tukey pairwise tests (Table 4) indicated highly significant improvements from the baseline interval (i.e., preimplantation status) to the first and second year postimplantation, respectively, for the HUI3 multiattribute utility scores. Results were similar for the SSQ subcategory scores, with a significant improvement also observed between baseline and the third year postimplantation for the group $(p<0.001)$. No significant differences were ob- served between other pairs of time points of evaluation $(p>0.057)$.

\section{Predictors of Longitudinal Outcomes}

The resulting $p$ values for the univariate analysis of the 12 preimplantation recipient variables (Table 1) with each outcome measure are presented in Table 5. Using the Bonferroni correction significance level, a significant impact upon outcomes was evident for 7 of the 12 variables examined ( $p \leq 0.004)$.

Recipients with comorbidities had significantly lower HUI3 scores than those without reported comorbidities (main effect, $p<0.001$ ). Similarly, those reporting dizziness or tinnitus prior to implantation had significantly lower HUI3 scores compared to those without either con-
68

Audiol Neurotol 2017;22:61-73 DOI: $10.1159 / 000477533$
Lenarz et al. 
dition (main effects, $p<0.001$ ). Recipients with preimplantation tinnitus had significantly lower mean SSQ speech and qualities scores (main effects, $p=0.002$ and $p=0.001$, respectively). A younger age at implantation (i.e., $<65$ years) resulted in significantly higher SSQ speech scores than for those aged $\geq 65$ years at implantation $(p=$ 0.004).

A significant interaction was shown between preimplantation contralateral hearing aid use and time for both the HUI3 multiattribute utility and SSQ qualities scores. Pairwise tests indicated that mean scores for both measures were significantly lower $(p=0.003$ and $p=0.004$, respectively) at baseline for recipients who did not wear a contralateral hearing aid than for those who did. Conversely, at 2 and 3 years postimplantation follow-up, mean HUI3 multiattribute utility scores were significantly higher for recipients who did not wear a contralateral hearing aid at preimplantation compared to those who did ( $p=0.010$ and $p=0.032$, respectively). Similarly, at 1 year postimplantation, SSQ qualities scores were significantly higher for recipients who did not use a contralateral hearing aid at preimplantation $(p=0.009)$.

A significant interaction between implant side and time for SSQ speech scores was observed $(p<0.002)$. While no difference was evident at baseline for SSQ speech ratings $(p=0.663)$, right-sided implantees scored significantly higher than left-sided implantes at each annual follow-up interval $(p<0.031)$. Telephone use had a significant association with all outcome measures at one or more time points. After adjusting for time, those who used the telephone had significantly higher mean SSQ speech scores, than those who did not $(p<0.001)$. For HUI3 outcomes, a difference was observed only at preimplantation, whereby those who used the telephone had significantly higher scores $(p<0.001)$ than nonusers. No impact of preimplantation telephone use was noted on HUI3 outcomes at other time points $(p>0.201)$. Similarly, for SSQ spatial and qualities scores, at preimplantation and at 1 year postimplantation assessment intervals, those who used the telephone at preimplantation had significantly higher scores $(p<0.032)$, while there was no significant impact thereafter $(p>0.102)$.

\section{Discussion}

The data presented in this study demonstrate the results of using the HUI3 and SSQ for longitudinal subjective assessment of hearing treatment outcomes in routine clinical practice in a multinational, multilingual, unilat-

Patient-Related Benefits for Adults with

Cochlear Implantation eral, primarily adult, CI recipient cohort. Longitudinal analysis demonstrated clinically and statistically highly significant patient-related benefits following unilateral CI treatment for both health-related quality of life and daily hearing ability for the study cohort via the HUI3 and the SSQ $(p<0.0001)$. Group means remained stable from 1 year postimplantation to 2 and 3 years postimplantation for all outcome measures. This is consistent with the reported daily CI use averaging $\sim 15 \mathrm{~h} /$ day at 2 years postimplantation, with no report of $<7 \mathrm{~h}$ per day observed.

\section{Impact of Missing Data}

Among the 291 enrolled unilateral CI recipients, 33\% of the subjects had missing data at one or more follow-up time points at the time of extraction. While there are no universally agreed upon criteria for acceptable follow-up rates in observational cohort studies, rates as high as $80 \%$ and as low as $60 \%$ (i.e., attrition rates between 20 and $40 \%$ ) have been suggested in the literature [Kristman et al., 2004]. There was no evidence of a statistically significant difference between outcomes for those with missing data at one or more follow-up time points compared to those with no missing data, suggesting that the missing data may be randomly missing. Upon closer examination of the reasons for the missing observations in our study, 10 out of 291 recipients were formally withdrawn early from this study by the enrolling clinic. The reasons provided included: death $(n=2)$, severe illness (non-hearing related) $(n=3)$, inability to complete forms reliably due to mental incapacity $(n=2)$, and reportedly being too busy to participate in follow-up appointments $(n=3)$. As an ongoing observational study, at the time of data extraction, the time since surgery ranged from just over 2 months to almost 5 years for the unilateral CI recipient cohort. Enrolled recipients with missing follow-up data $\geq 18$ months after their surgery date were deemed as lost to follow-up $(n=37)$, while data entry was considered still likely for enrolled recipients with $<18$ months of CI experience $(n=49)$. This is based on the observation that online data entry at follow-up intervals was delayed by up to several months after completion of the clinical assessment in some cases in view of the clinical time constraints for performance of the online data entry. Hence the missing data indicates that the actual subject attrition rate for our study could be as high as $33 \%$ or as low as $17 \%$.

\section{Cohort}

The study cohort characteristics are similar to those reported in the literature for adult unilateral CI recipients (only a handful of adolescents were enrolled, consistent

Audiol Neurotol 2017;22:61-73

DOI: $10.1159 / 000477533$ 
with infrequent implantation in new recipients at that age). Implanted adult CI groups are often in their early 50 s on average, with a wide age range, a mean duration of deafness of close to 2 decades, bilateral deafness with a severe to profound degree of loss at minimum and most often a profound hearing loss, and presentation of a wide range of etiologies, with the most common reported as unknown by researchers [UK Cochlear Implant Study Group, 2004; Damen et al., 2007; Maes et al., 2011].

\section{Country Effect}

The collation of multicenter, multinational data sets for group analysis represents outcomes for a variety of clinical infrastructures and socioeconomic and cultural settings in which hearing treatment with a CI is provided. Analysis of the effect of country of origin on the data sets did not show an effect on outcomes for the HUI3 multiattribute utility scores over time, although the main effect was in fact borderline nonsignificant. Researchers who compared outcomes from a validated Spanish translation version of the HUI3 to the English version (i.e., 15 questions with a 4 -week recall) within a national cross-sectional population sample, with differing ethnicity, social, and living conditions, reported that they observed a significant cultural effect [Lou et al., 2009]. They hypothesized that the level of optimism, confidence, and willingness to report on health issues may be ethnicity specific and, thus, impact the overall health status report.

Our study confirmed a significant country effect for SSQ subcategory scores $(p<0.02)$, which resulted in incorporation of a random intercept for the variable country into the subsequent analysis of SSQ scores to control for these country-specific differences. This finding is consistent with that reported by researchers developing and assessing the validated translation of the SSQ French version, following comparison of their data gathered on normal-hearing and hearing-impaired (nonimplanted) French individuals, to published mean data derived from hearing-impaired Dutch and English populations [Moulin et al., 2015]. They concluded by recommending that the SSQ is considered a feasible international standard assessment for use clinically.

Based on our study findings, our recommendation is to assess for country effects when examining aggregated multicultural cohort data for SSQ and HUI3 outcomes to ensure accountability of significant effects via a mixedeffects model for further analysis as needed. Furthermore, further examination of additional recipient characteristics, such as educational level, social activity, living status and ethnicity, may also be beneficial to collate for subse- quent analysis of their association with outcomes on HUI3 and SSQ subjective measures.

\section{Longitudinal HUI3 Outcomes}

Our study suggests that the HUI3 is a sensitive measure of the changes in overall health following hearing treatment over time for CI users cross-culturally. Furthermore, as it is a quick and easy self-administered tool to use, it is feasible to consider, as part of routine clinical assessment, for systematic evaluation of the broader patient-related benefits of hearing implant treatment.

Individual analysis suggests that clinically significant improvements from baseline were observed by about two thirds of the recipient group, which remained stable at 1 , 2 , and 3 years postimplantation, while $25 \%(8 / 32)$ of the individuals with 3-year data showed a decline in HUI3 scores from baseline. Upon examination, no differences in characteristics were evident between recipients who showed a decline and those who remained stable. Both subgroups were primarily under 65 years of age, employed, and without reported comorbidities.

Also of interest is the subgroup of 6 individuals who reported a state "worse than dead" at the 1-year follow-up; 1 out of 6 patients also reported the negative state at baseline, while no such reports were observed at the most recent follow-up at 2 years postimplantation (Fig. 2d). All 6 recipients were female, had a mean age of 55.5 years (SD 11.9 , range 35-70), four reported severe pain via the HUI3 at the 1-year follow-up while the remaining two reported poor to severe impairment on at least 2 other domains. While the group is too small to make a firm conclusion, poor ratings on one or more health domains have the potential to negatively influence the overall HUI3 multiattribute health utility score for the individual.

\section{Predictors of HUI3 Outcomes}

For our study cohort differences observed for HUI3 outcomes were associated with preimplantation report of comorbidities, dizziness, tinnitus, telephone use, and contralateral hearing aid use. Using the stringent significance level of $p=0.004$, we found no association between age at implantation or duration of hearing loss and HUI3 outcomes. The absence of an age effect on HUI3 outcomes was also reported by researchers from a multicenter, national longitudinal study on unilateral CI recipients; however, they did observe an association with a longer duration of hearing loss (i.e., $\geq 40$ years) [UK Cochlear Implant Study Group, 2004]. In contrast, researchers reporting on a long-term comparative study involving a small group of unilateral CI recipients with several years
70

Audiol Neurotol 2017;22:61-73

DOI: $10.1159 / 000477533$
Lenarz et al. 
of CI experience observed a significant age effect and suggested a possible influence of age-related comorbidities with increasing time [Damen et al., 2007].

For future longitudinal studies, it may be of interest to collate more detailed information about changes in the severity of reported comorbidities, including tinnitus and dizziness, in general over time and to examine their association with subjective outcomes.

\section{Longitudinal SSQ Outcomes}

Results from repeated measures on the SSQ suggest significant improvement from preimplantation scores. The mean change from baseline (preimplantation status) to 1 year postimplantation ranged from 2.1 to 2.4 across SSQ subcategories and were notably well above the clinically significant change reported by the developers of the SSQ, who suggest that a 1.0-scale-point difference is typical after providing a unilateral HA to hearing impaired adult groups [Noble and Gatehouse, 2004].

An individual analysis of outcomes within our study group suggested that $70 \%$ of the individuals showed significant improvement at 1 year postimplantation on one or more SSQ subcategories relative to baseline ratings. The proportion of subjects with a clinically significant change in SSQ scores increased to as high as $90 \%$ over time, while there were no subjects for any SSQ subcategory who had a clinically meaningful decrease from baseline to 3 years postimplantation. For some subjects, improvement in self-assessed ratings of hearing function continued beyond the first year postimplantation.

As pointed out by researchers such as Mertens et al. [2015], auditory disability cannot be measured or reliably predicted based on laboratory testing of speech outcomes alone, but real-world measures such as questionnaires like the SSQ should be included to assess the preimplantation and posttreatment effects on hearing function over time.

\section{Predictors of SSQ Outcomes}

In our study cohort, SSQ subcategory ratings were shown to differ between several covariate subgroups for the variables examined. As shown in Table 5, preimplantation status, such as contralateral HA use, being younger than 65 years of age, implant side, and the ability to use the telephone, was associated with significantly higher scores on one or more of the SSQ subcategories at one or more of the assessment time points. In contrast, the report of tinnitus at preimplantation had a significant negative effect for SSQ speech and SSQ qualities scores over time ( $p=0.002$ and $p=0.001$, respectively).

Patient-Related Benefits for Adults with Cochlear Implantation
In a recent publication, examination of the outcomes on the SSQ for a group of nonimplanted hearing-impaired subjects versus normal-hearing subjects suggested that the main predictive effects of outcomes were in association with hearing loss degree in the better ear and interaural hearing loss asymmetry [Moulin and Richard, 2016]. As has also been asuggested by other researchers, closer examination of the degree of interaural asymmetry, including the subsequent classification of the contralateral ear as either the better, the poorer or an equivalent hearing ear, may be relevant to further assess the impact on outcomes on the SSQ [UK Cochlear Implant Study Group, 2004; Noble and Gatehouse, 2006; Noble et al., 2008].

\section{Study Design Limitations}

While this was an observational study, with investigators instructed to offer participation to consecutively implanted adult and adolescent Nucleus ${ }^{\circledR}$ implant recipients treated in routine practice, the potential for enrolment bias cannot be excluded. Investigator motivation and time to participate may vary and influence the approach towards newly implanted recipients for study participation. Inherent self-selection bias by the recipient may also exist. For example, recipients willing to participate may be inherently more motivated, active, or positive in reporting their outcomes than those who decline to participate. It is also possible that the willingness and agreement to participate are influenced by the recipient's levels of anxiety and or pain related to upcoming or recent surgical therapy. Furthermore, as the study design requires reading skills to complete self-assessments of outcomes, those unable to read are less likely to be enrolled. as are recipients with severe comorbidities.

Hence, while attempts are made to reduce possible enrolment bias as much as possible, caution should be exercised when attempting to generalize the findings of this study beyond the study cohort to the treatment population at large, without further exploration of the population characteristics of the enrolled cohort versus the nonenrolled implant recipient group at each participating site.

The observational study design used calls for a record of baseline evaluations between the day of the surgery and the first fitting, with a recall time reference of " 4 weeks prior to implantation." A potential bias of preimplantation status evaluation may occur as a result of the condition of the recipient during the postsurgery healing phase, with possible pain or discomfort and the absence of auditory stimulation in the implanted ear. Furthermore, as some recipients may have been asked to assess their preimplantation status immediately before the first fitting of

Audiol Neurotol 2017;22:61-73

DOI: $10.1159 / 000477533$ 
their sound processor, this may have extended the recall period for the 4 -week reference interval by up to 2 or 3 months. Nonetheless, as the preimplantation reference interval is marked by a significant milestone, the implant surgery, the degree of recall bias, specifically in relation to health status, is expected to be minimized [Barsky, 2002].

As the numbers of available data sets were reduced at each subsequent postimplantation interval, repeating the analysis of all available data for larger numbers of enrolled CI recipients may be of benefit to further examine changes in outcomes over time and the influencing factors.

\section{Conclusions}

Our collaborative, multinational observational study of longitudinal patient-related outcomes for a cohort of routinely treated unilateral CI recipients evaluated via standardized self-assessment measures, i.e., the HUI3 and SSQ, demonstrated significant improvements in health-related quality of life and real-life hearing function following implantation for the group.

Mean improvements remained relatively stable beyond 1 year postimplantation for the available data sets at 2 and 3 years postimplantation. In view of the multicultural population, examination and consideration of differences between countries for each outcome used are recommended, with adaptation of the longitudinal models used as appropriate. Changes in outcome measures were significantly associated with recipient characteristics as possible predictors of performance over time. Specifically, associations with one or more outcome measures were noted for the following preimplantation variables: age at implantation, implantation side, telephone use, contralateral hearing aid use, and additional comorbidities.

The subjective outcome measures used in our study design provide a holistic view of the real-life treatment benefits of routine CI therapy for adult recipients. Furthermore the measures used may be feasible for routine clinical use and enable aggregation of cross-cultural data sets as medical evidence to support decisions by health professionals and service providers.

\section{Acknowledgements}

The coauthors would like to sincerely acknowledge the contributions of the following academics and experts at each clinic for their support in working with the implant recipients for counsel- ing for their study participation, performing evaluations, and data capture. In alphabetical order by implantation city or clinic: Andrzej Sieśkiewicz and Marek Rogowski from Bialystok, Poland; Andreas Buechner, Anke Lesinski-Schiedat, Hannes Maier, Eugen Kludt, and Angelika Illg from Hannover, Germany; Rozario Lezaun from Pamplona, Spain; Bożena Wiskirska-Woźnica, Alicja Sekula, and Magdalena Kałos from Poznan, Poland; Maria del Prado Venegas Pizarro and Julia de Juan Beltran from San Sebastian, Spain; Jennifer Perold, Gill Kerr, Suryn Lombaard, Deidré Stroebel, and Surida Booysen from Tygerberg, South Africa; Juan Royo Lopez from Zaragoza, Spain; and Sara de Torres Iera and Lara Roman Delgado from GAES (Spain).

\section{Disclosure Statement}

The authors declare that they have no conflict of interests regarding the observational trial participation. The sponsor of this observational study, i.e., Cochlear AG, funded and developed the Cochlear IROS e-platform, which it maintains via external consultant data base developers and hosts, Lambda Plus. Participation in the study was voluntary and the e-platform is a free service offered for voluntary participation to implant clinics and it is available for data collection for recipients of any hearing implant from Cochlear. J.W. and C.I. are employees of Cochlear AG. The consultant statistician, P.L.G, a coauthor of this paper, performed the statistical analysis of the data and was funded by Cochlear AG for her work. No payment was provided to recipients as study participants.

\section{Funding Sources}

The Cochlear IROS is a commercially funded, international observational study of patient-related outcomes for recipients of any commercially available implant from the company Cochlear. Participation is on a voluntary basis for implant clinics and implant recipients. The sponsor of this study, Cochlear AG, does pay clinic investigators or patients for their participation.

\section{Author Contributions}

The coauthors T.L., L.M., H.C.-W., H.V.V., C.O.D., M.D., A.H.I., B.P., and M.Z., as clinical investigators, were actively involved in the conduction of this study in their respective clinics. C.I. is a Cochlear employee and was involved in the preliminary descriptive report of the full data set. P.L.G., a consultant statistician from Macquarie University, was responsible for guiding, performing, and describing the statistical analysis. J.W. is an employee of Cochlear and was responsible for the design and implementation of this study and was the main writer of this report. All of the coauthors reviewed and approved this paper for submission.
72
Audiol Neurotol 2017;22:61-73

DOI: $10.1159 / 000477533$
Lenarz et al. 


\section{References}

Barsky AJ: Forgetting, fabricating, and telescoping: the instability of medical history. Arch Intern Med 2002;162:1-4.

Berrettini S, Arslan E, Baggiani A, Burdo S, Cassandro E, Cuda D, Dinelli E, Filipo R, Mancini P, Martini A, Quaranta A, Quaranta N, Turchetti G, Forli F: A registry for the collection of data in cochlear implant patients. Acta Otorhinolaryngol Ital 2011;31:328-340.

Brand Y, Senn P, Kompis M, Diller N, Allum J: Cochlear implantation in children and adults in Switzerland. Swiss Med Wkly 2014;144: w13909.

Damen GW, Beynon AJ, Krabbe PF, Mulder JJ, Mylanus EA: Cochlear implantation and quality of life in postlingually deaf adults: long-term follow-up. Otolaryngol Head Neck 2007;136:597-604.

Feeny D, Furlong W, Boyle M, Torrance GW: Multi-attribute health status classification systems: Health Utilities Index. Pharmacoeconomics 1995;7:490-502.

Feeny D, Furlong W, Torrance GW, Goldsmith $\mathrm{CH}$, Zhu Z, DePauw S, Denton M, Boyle M: Multi-attribute and single-attribute utility functions for the Health Utilities Index Mark 3 system. Med Care 2002;40:113-128.

Gatehouse S, Noble W: The Speech, Spatial and Qualities of Hearing Scale (SSQ). Int J Audiol 2004;43:85-99.

Gliklich RE, Dreyer NA: Registries for Evaluating Patient Outcomes: a User's Guide, ed 2. Rockville, Agency for Healthcare Research and Quality, 2010.

Haute Autorité de Santé: Choix méthodologiques pour l'évaluation économique à la HAS. 2011. http://www.has-sante.fr/portail/jcms/ r_1499251/en/choix-methodologiques-pourl-evaluation-economique-a-la-has (accessed May 2014).

Health Utilities Incorporated: Health-related quality of life. www.healthutilities.com (accessed January 2011).

House JW, Kutz JW Jr, Chung J, Fisher LM: Boneanchored hearing aid subjective benefit for unilateral deafness. Laryngoscope 2010;120: 601-607.

International Organization for Standardization: ISO 14155:2011: clinical investigation of medical devices for human subjects - good clinical practice. 2011. http://www.iso.org/ iso/catalogue_detail?csnumber $=45557 \quad$ (accessed December 2011).
Kristman V, Manno M, Cote P: Loss to follow-up in cohort studies: how much is too much? Eur J Epidemiol 2004;19:751-760.

Laske RD, Veraguth D, Dillier N, Binkert A, Holzmann D, Huber AM: Subjective and objective results after bilateral cochlear implantation in adults. Otol Neurotol 2009;30:313318.

Little RJA, Rubin DB: Statistical analysis with missing data. Hoboken, Wiley, 2002.

Lou N, Ko Y, Johnson JA, Coons SJ: The association of survey language (Spanish and English) with Health Utilities Index and EQ-5D index scores in a United States population sample. Qual Life Res 2009;18:1377-1385.

Maes IH, Joore MA, Cima RF, Vlaeyen JW, Anteunis LJ: Assessment of health state in patients with tinnitus: a comparison of the EQ5D and HUI Mark III. Ear Hear 2011;32:428435.

Mertens G, Kleine Punte A, De Bodt M, Van de Heyning P: Binaural auditory outcomes in patients with postlingual profound unilateral hearing loss: 3 years after cochlear implantation. Audiol Neurootol 2015;20(suppl 1):6772.

Moulin A, Pauzie A, Richard C: Validation of a French translation of the Speech, Spatial, and Qualities of Hearing scale (SSQ) and comparison with other language versions. Int $\mathrm{J} \mathrm{Au}-$ diol 2015;54:889-898.

Moulin A, Richard C: Sources of variability of Speech, Spatial and Qualities of Hearing scale (SSQ) scores in normal-hearing and hearingimpaired populations. Int J Audiol 2016;55: 101-109.

National Institute for Health and Clinical Excellence: Cochlear implants for children and adults with severe to profound deafness: technology appraisal guidance - TA1066. 2009. http://www.nice.org.uk/guidance/ta166 (accessed September 2015).

National Institute for Health and Clinical Excellence: Guide to the methods of technology appraisal. 2013. http://publications.nice.org.uk/ pmg9 (accessed May 20, 2014).

Noble W: Assessing binaural hearing: results using the speech, spatial and qualities of hearing scale. J Am Acad Audiol 2010;21:568-574.
Noble W, Gatehouse S: Interaural asymmetry of hearing loss, Speech, Spatial and Qualities of Hearing scale (SSQ) disabilities, and handicap. Int J Audiol 2004;43:100-114.

Noble W, Gatehouse S: Effects of bilateral versus unilateral hearing aid fitting on abilities measured by the Speech, Spatial, and Qualities of Hearing scale (SSQ). Int J Audiol 2006;45: 172-181.

Noble W, Tyler R, Dunn C, Bhullar N: Unilateral and bilateral cochlear implants and the implant-plus-hearing-aid profile: comparing self-assessed and measured abilities. Int J Audiol 2008;47:505-514.

Pai I, Kelleher C, Nunn T, Pathak N, Jindal M, O'Connor AF, Jiang D: Outcome of bone-anchored hearing aids for single-sided deafness: a prospective study. Acta Otolaryngol 2012; 132:751-755.

R Core Team: R: a language and environment for statistical computing. 2015. https://www.Rproject.org/ (accessed June 2015).

Tufts Evidence-Based Practice Centre under contract by the Agency for Health Research and Quality: Effectiveness of cochlear implants in adults with sensorineural hearing loss: health technology report - project ID AUDT0510. 2011. https://www.cms.gov/Medicare/Coverage/DeterminationProcess/downloads/id80TA.pdf (accessed December 2012).

UK Cochlear Implant Study Group: Criteria of candidacy for unilateral cochlear implantation in postlingually deafened adults. 1 . Theory and measures of effectiveness. Ear Hear 2004;25:310-335.

US Food and Drug Administration: Challenges for device postmarket surveillance. 2013a. http://www.fda.gov/MedicalDevices/Safety/ CDRHPostmarketSurveillance/ucm 348792. htm.

US Food and Drug Administration: Strengthening our national system for medical device post-market surveillance. 2013b. http://www. fda.gov/downloads/MedicalDevices/Safety/ CDRHPostmarketSurveillance/UCM348845. pdf.

World Medical Association: Declaration of Helsinki: ethical principles for medical research involving human subjects - 64th WMA General Assembly, Fortaleza, Brazil. 2013. https:// www.wma.net/policies-post/wma-declaration-of-helsinki-ethical-principles-for-medical-research-involving-human-subjects/ (accessed October 2013).
Patient-Related Benefits for Adults with Cochlear Implantation
Audiol Neurotol 2017;22:61-73

DOI: $10.1159 / 000477533$ 